\title{
PERCEPCIONES DE PERSONAS MAYORES SOBRE SU DESEMPEÑO EN EL USO DE TRANSPORTE PÚBLICO EN SANTIAGO DE CHILE
}

\section{PERCEPTIONS OF OLDER PEOPLE ON THEIR PERFORMANCE IN THE USE OF PUBLIC TRANSPORTATION IN SANTIAGO, CHILE}

\author{
Gajardo J. ${ }^{1}$, Navarrete E,. ${ }^{2}$, López C. ${ }^{3}$, Rodríguez J. ${ }^{3}$, Rojas A. ${ }^{3}$, Rojas A ${ }^{3}$, Troncoso S. ${ }^{3}$
}

\begin{abstract}
RESUMEN
Justificación: la movilidad en la comunidad es esencial en la vida de toda persona. El uso de transporte es una actividad instrumental que posibilita la movilidad en la comunidad. En la vejez esta actividad puede presentar características asociables al envejecimiento individual, la actividad misma, y el ambiente. Emerge la relevancia de conocer esta ocupación en las personas mayores chilenas y su implicancia para la Terapia Ocupacional como disciplina promotora de participación y autonomía en la vejez. Objetivo: se buscó explorar características del desempeño de un grupo de personas mayores en el uso transporte en Santiago a partir de sus experiencias y percepciones. Metodología: Se realizó diez entrevistas a adultos mayores que luego fueron analizadas con un enfoque interpretativo - hermenéutico. Resultados: Cinco temas principales emergieron del análisis de entrevistas: "función del uso del transporte público", "adaptaciones preparatorias para un mejor desempeño", "influencia del ambiente”, “¿bus o metro? razones para la elección” y "significado del uso: autonomía versus exclusión". Conclusiones: En el grupo participante el uso del transporte se veía limitado por el ambiente físico, y facilitado por el ambiente social, y las personas desplegaban estrategias adaptativas para un mejor desempeño. El significado del uso de transporte se relacionó con la mantención de la autonomía y la identidad. El sistema de transporte en Santiago puede no ajustarse a las necesidades de la población mayor y puede percibirse como un elemento de exclusión social para las personas mayores.
\end{abstract}

Palabras Clave: vejez, transporte público, desempeño ocupacional, movilidad en la comunidad.

\begin{abstract}
Justification: community mobility is essential for daily living. The use of transportation is an instrumental activity of daily living that facilitates community mobility. In the elderly this activity may be conditioned by features related to the aging process, the activity itself, and the environment in which it is carried out. The importance of learning about the characteristics of this occupation in Chilean older people emerges as well as implications for occupational therapy as a promoter of social participation and autonomy in old age. Objective: to explore performance characteristics of a group of older people in the use of public transportation system in the city of Santiago. Methods: Ten interviews were conducted to older adults. Interviews were later interpretively analyzed from a hermeneutic approach. Results: Five main themes emerged from the speeches of the participants, "function of the use of public transportation", "preparatory adaptations for better performance," "environmental influence", "bus or subway? Reasons for this choice" and "meaning of the use of transport: autonomy versus exclusion." Conclusions: In the group of participants the use of transport is interfered by the physical environment, and facilitated by the social environment, and adaptive strategies are deployed. The meaning of the activity

\footnotetext{
${ }^{1}$ Terapeuta Ocupacional, Máster en Gerontología Social, Licenciado en ciencias de la Ocupación Humana. Profesor Asistente Escuela de Terapia Ocupacional Universidad de Chile. Independencia 1027, Santiago. tel: +5629786342, jgajardo@med.uchile.cl

${ }^{2}$ Terapeuta Ocupacional, Master in Medical Sciences in Occupational Therapy, Licenciada en Ciencias de la Ocupación Humana. Profesor Asistente Escuela de Terapia Ocupacional Universidad de Chile. Independencia 1027, Santiago. +5629786587 , enavarre@med.uchile.cl

${ }^{3}$ Terapeuta Ocupacional, Licenciada en Ciencias de la Ocupación Humana, Universidad de Chile.
} 
relates to the maintenance of autonomy and identity. The transport system in Santiago may not fit the needs of older people and may be perceived as an element of social exclusion in the elderly.

Key words: Old age, public transportation, occupational performance, community mobility.

\section{INTRODUCCIÓN}

La Encuesta de Caracterización Socioeconómica Nacional (CASEN) estimó que en el año 2006 el 13\% de la población chilena tenía 60 años o más (Ministerio de Planificación, 2006). Se espera que el número de personas mayores siga en aumento, proyectándose que en el año 2020 un $17 \%$ de la población total en Chile tendrá una edad de 60 años o más (Instituto Nacional de Estadísticas, 2002), reflejando el progresivo envejecimiento demográfico del país. Una mayor longevidad de la población implica una mayor prevalencia de enfermedades crónicas y degenerativas que pueden llevar a diferentes grados de limitación funcional, física, mental y social, con la consiguiente necesidad de ayuda para la realización de las actividades de la vida diaria que son fundamentales para el bienestar individual (Duarte \& Pedro, 2010). En relación a esto, en el año 2009 se estimó que cerca del $80 \%$ de las personas mayores chilenas son independientes en actividades de la vida diaria básicas e instrumentales (Servicio Nacional del Adulto Mayor, 2009). En este contexto emerge el desafío para la Terapia Ocupacional de conocer los factores relacionados con el desempeño ocupacional de las personas mayores en las actividades diarias, en pos de colaborar con la promoción de un desempeño ocupacional satisfactorio y 
autónomo que se mantenga por el mayor tiempo posible. El concepto desempeño ocupacional se define como:

"la capacidad de elegir, organizar y desempeñar de manera satisfactoria ocupaciones significativas definidas por la cultura y adecuadas a la edad, el cual comprende a la persona, su ambiente concebido como los contextos y situaciones que tienen lugar fuera de los sujetos; y la ocupación, descrita como el estudio de la forma, función y significado de la actividad (Kielhofner 2006, p. 96)

Un aspecto de relevancia en la mantención de la independencia y la autonomía de toda persona es el de la movilidad en la comunidad, definida por la Asociación Americana de Terapia Ocupacional (2008) como:

"moverse por la comunidad y utilizar el transporte público o privado, como conducir, caminar, andar en bicicleta, o acceder al autobús, taxi u otros sistemas de transporte".

Como se observa, el uso de transporte público corresponde a una actividad instrumental para la movilidad en la comunidad. En su relación con la vejez, el uso del transporte público es considerado una herramienta fundamental para la integración social de las personas mayores en la comunidad, su mantención de la autonomía y el cuidado de la salud mental de la población mayor (Ramos \& Nazif, 2005). No obstante lo anterior, el envejecimiento puede impactar en la capacidad de la persona mayor para usar el transporte generándose dificultades y propiciando escenarios para el potencial desarrollo de estrategias individuales para su optimización (Wallace \& Franc, 1999).

En nuestro país se ha generado conocimiento sobre la interacción de los adultos mayores chilenos con el transporte público terrestre, evidenciando diversas dificultades en términos de seguridad y conocimiento del funcionamiento del sistema, y así mismo, se ha sentado bases sobre la relevancia del uso de transporte público terrestre para las personas mayores como una actividad que promueve la mantención del vínculo con la comunidad (Ramos \& Nazif, 2005).

Se ha recomendado que la planificación de los sistemas de transporte, y otras fuentes de movilidad, incorporen información proveniente de investigación basada en las percepciones de las personas respecto al acceso, la aceptabilidad, la utilidad, las necesidades y deseos personales, para una mayor eficacia (Wallace \& Franc, 1999; US transportation research Board Council, 1998). A partir de esto sobresale la importancia de profundizar conocimientos en esta área de la ocupación ya que se posiciona como un área importante para apoyar la vida diaria dentro de la comunidad de las personas mayores.

En el contexto de cambio que ha experimentado durante los últimos años el sistema de transporte terrestre de Santiago ${ }^{4}$, este trabajo tuvo como propósito explorar la percepción de un grupo de personas mayores sobre su desempeño en el uso del transporte público de esta ciudad. De este modo, los objetivos específicos que se desprendieron buscaron indagar, según fue definido con anterioridad, en los componentes específicos del desempeño atribuibles a la persona, al ambiente y/o a la ocupación misma.

${ }^{4}$ El sistema actual de transporte público de Santiago se denomina "Transantiago", y fue implantado de manera definitiva en el año 2007. Considera cambios en los mecanismos de pago, recorridos, y tipología de los dispositivos de transporte. 
Para efectos de esta investigación, el concepto de movilidad en la comunidad se remitió al uso de buses urbanos y metro, ya que el actual sistema de transporte público de Santiago combina en su funcionamiento ambos dispositivos, además de la integración de un sistema de pago de tarifa a través de una tarjeta electrónica y de la incorporación de nuevas rutas y paraderos (Ministerio de Transportes y Telecomunicaciones, 2012).

\section{METODO}

Para el logro de los objetivos se utilizó una metodología de tipo cualitativa, la cual permite estudiar la realidad en su contexto habitual, intentando extraer sentido o interpretar los fenómenos de acuerdo con los significados que tienen para las personas implicadas (Rodríguez, Gil \& García, 1996). Para la recolección de datos se utilizó una entrevista en profundidad que buscó rescatar la percepción de las personas mayores en el uso del transporte público, según sus experiencias y buscando la emergencia de temas según la relevancia percibida por el o la entrevistado/a. Para fines metodológicos se preparó un guión de temas y preguntas para la entrevista, el que antes de su aplicación, fue sometida a prueba con personas mayores no incluidas en el grupo de participantes. Esto permitió eliminar o reformular preguntas y temas del guión de entrevista inicial.

La ejecución de las entrevistas se dio entre los meses de abril y mayo del año 2011, con una duración por entrevista de aproximadamente una hora, las cuales fueron grabadas.

Esta investigación buscó explorar cómo los y las participantes percibían sus experiencias en el uso del transporte público Transantiago, considerando el concepto de percepción como un proceso cognitivo que consiste en el reconocimiento, la interpretación y significación de sensaciones obtenidas del ambiente físico y social, para la elaboración de juicios al respecto (Vargas, 1994). A su vez, para el análisis e interpretación de datos se utilizó un enfoque hermenéutico, propio de los procesos cualitativos, el cual tiene como característica interpretar y comprender para desvelar los motivos del actuar humano (Aristizábal, 2008). El presente estudio de tipo exploratorio, buscó favorecer el acercamiento a un fenómeno escasamente estudiado en nuestro país, a partir de la percepción de las personas mayores sobre su desempeño en el uso del transporte público.

La selección de los participantes se realizó por conveniencia y los criterios de inclusión fueron:

- Tener 60 años o más de edad,

- No depender de otra persona en la utilización del transporte,

- No tener diagnóstico de deterioro cognitivo, y

- Utilizar el transporte público con una frecuencia mínima de cuatro veces al mes.

Los participantes del estudio fueron contactados por medio de dos instituciones: la Sección de Geriatría del Hospital Clínico de la Universidad de Chile y la Fundación Villa Padre Alberto Hurtado, con quienes se estableció contacto a través del envío de una carta formal a los directores de cada establecimiento, solicitando la colaboración directa de sus equipos para el reclutamiento de potenciales participantes que cumplieran con los requisitos de ingreso a través de una lista de cotejo. A medida que eran referidos, los potenciales participantes eran contactados de manera presencial o vía telefónica por un representante del equipo investigador con el fin de: corroborar el cumplimiento de los criterios de 
inclusión, explicar los objetivos y metodologías de la investigación y aspectos concretos de su potencial participación e invitar a participar en la investigación. Si la respuesta era positiva, se procedía a planificar un encuentro personal posterior entre el investigador y el participante para realizar la entrevista. El lugar de la reunión buscó concretarse en un ambiente cotidiano para el/la participante, por lo que se realizó en el domicilio personal de cada uno.

El grupo de participantes se constituyó por diez personas (4 hombres y 6 mujeres, promedio etario de 77 años, rango de 68 a 88 años) cuyos datos generales se refieren en la Tabla 1.

Tabla 1. Datos generales de los participantes

\begin{tabular}{|c|c|c|c|c|c|c|}
\hline Participante & Mujer & Hombre & $\begin{array}{c}\text { Edad } \\
\text { (años) }\end{array}$ & $\begin{array}{c}\text { Estado } \\
\text { civil }\end{array}$ & $\begin{array}{c}\text { Comuna de } \\
\text { residencia }\end{array}$ & $\begin{array}{c}\text { Realiza } \\
\text { actividad } \\
\text { productiva }\end{array}$ \\
\hline EJ & $\mathrm{x}$ & & 80 & Viuda & Recoleta & \\
\hline AA & $\mathrm{x}$ & & 75 & Casada & Renca & $\mathrm{X}$ \\
\hline JV & & $\mathrm{x}$ & 68 & Soltero & $\begin{array}{c}\text { Pedro Aguirre } \\
\text { Cerda }\end{array}$ & \\
\hline LG & $\mathrm{x}$ & & 74 & Viuda & San Miguel & \\
\hline AG & $\mathrm{x}$ & & 69 & Casada & $\begin{array}{c}\text { Pedro Aguirre } \\
\text { Cerda }\end{array}$ & \\
\hline OC & $\mathrm{x}$ & & 79 & Soltera & Independencia & \\
\hline RL & $\mathrm{x}$ & & 74 & Soltera & $\begin{array}{c}\text { Pedro Aguirre } \\
\text { Cerda }\end{array}$ & \\
\hline MR & & $\mathrm{x}$ & 88 & Casado & $\begin{array}{c}\text { Pedro Aguirre } \\
\text { Cerda }\end{array}$ & \\
\hline EG & & $\mathrm{x}$ & 79 & Casado & Recoleta & \\
\hline JL & & $\mathrm{x}$ & 86 & Viudo & Conchalí & \\
\hline
\end{tabular}

El encuadre de entrevista consideró tres etapas: explicitación de objetivos de investigación y de temas y preguntas a realizar; lectura conjunta de consentimiento informado y libre firma de éste; y finalmente la entrevista. La información entregada tuvo el propósito de asegurar que la persona contara con todos los antecedentes necesarios y suficientes para decidir autónomamente su participación por medio de la firma del consentimiento informado.

Posteriormente las entrevistas fueron transcritas en formato word, para su análisis cualitativo. Siguiendo las recomendaciones de Huber y Gürtler (2008), se realizó una codificación que permitió la reducción de la información y posteriormente a través del análisis de contenidos se generó categorías iniciales, trece en este caso. Luego se realizó en dos oportunidades el refinamiento y reducción de dichas categorías iniciales, con el fin de rescatar aspectos significativos del desempeño ocupacional y sus componentes persona, ambiente, y ocupación; y ésta última caracterizada por la forma, función y significado, según se refirió anteriormente. De estas trece categorías emergieron 5 temas principales, los que entregan información sobre el desempeño de los participantes en el uso del transporte público en Santiago. Los principales temas se enumeran a continuación:

1. Función del uso del transporte público 
2. Adaptaciones preparatorias para un mejor desempeño

3. Influencia del ambiente

4. ¿Bus o metro? Razones para la elección.

5. Significado del uso: autonomía versus exclusión

\section{Aspectos bioéticos}

La participación en este estudio no significó riesgo físico, psíquico ni moral para las personas implicadas. Todos los participantes firmaron consentimiento antes de ser entrevistados. Los propósitos del consentimiento informado fueron: verificar la comprensión de la información entregada en relación con los objetivos de la investigación y las características de su participación, explicitar la libertad de la participación, y autorizar al equipo de investigadores para usar la información obtenida con fines académicos y científicos resguardando la confidencialidad de los datos personales entregados.

Los objetivos de esta investigación se relacionaron principalmente con los principios bioéticos de beneficencia y justicia, en la medida que buscó generar información que pueda aportar a un mejor conocimiento de las experiencias de personas mayores en el uso del transporte público, y por ende colaborar en la toma de decisiones para beneficiar a este grupo etario.

\section{RESULTADOS}

A partir del análisis de las entrevistas emergieron cinco temas principales, los que entregan información sobre el desempeño de los participantes en el uso del transporte público en Santiago. A continuación se describe en mayor profundidad los principales contenidos de cada unidad temática.

\section{La función del transporte público}

Los participantes utilizaban el transporte público entre dos y cinco veces a la semana, y realizaban recorridos que abarcaban más allá de la comuna en que viven, lo que en ocasiones incluía combinaciones entre metro y bus. Los participantes utilizaban el transporte público para asistir a actividades sociales junto a familiares, amigos y/o instancias comunitarias, así como para realizar compras. Otros lo utilizaban como medio de movilización para pagar cuentas o cobrar sus pensiones.

De este modo, el uso del transporte público emergía como una actividad instrumental facilitadora de la realización de otras actividades instrumentales o de participación social. 


\section{Adaptaciones preparatorias para un mejor desempeño}

Este tema incorpora diversas modificaciones que las personas realizaban para facilitar y/o permitir el desarrollo de la actividad. Una de estas adaptaciones consistía en el preocuparse de conocer a cabalidad las rutas frecuentes, estudiarlas previamente, y evitar modificarlas, con el objetivo de evitar la improvisación que pudiese llevar a error o riesgo.

En relación con el acceso a información y la preparación de los viajes, los participantes refirieron que para acceder a información relativa al sistema Transantiago consultaban a conocidos, familiares y/o funcionarios directos de los distintos dispositivos bus/metro. Del mismo modo, valoraban el uso de planos ubicados en las estaciones del metro, así como la información entregada por medios de comunicación masiva y mediante planos personales que algunos utilizaban durante sus viajes.

Los participantes refirieron modificar sus patrones temporales de uso del transporte público para un mejor desempeño. Se identificó una preferencia de uso matinal, específicamente entre las 10:00 y 11:00, ya fuese en metro o en bus. Según fue referido: “...trato de no irme a esas horas de mucho ajetreo (horas pico), que va demasiado lleno...". Para facilitar esto, algunos participantes organizaban sus rutinas con anticipación con el fin de evitar los horarios de mayor afluencia, según lo comentado: “...si tengo que pedir hora al médico y me dicen a las ocho, no la tomo. Prefiero un horario en que yo pueda tomar bien el metro...". Esta adaptación de patrones temporales, decía también relación con poder utilizar el beneficio descuento para la persona mayor en el dispositivo metro, válido de 9:00 a 18:00 horas. La adaptación de patrones temporales también emergió asociado con la percepción de un mayor riesgo durante la noche, según fue referido: “... si me veo obligado a regresar tarde pido un taxi siempre (...) tengo miedo que me asalten...”.

Otra adaptación que buscaba un mejor desempeño, tuvo relación con el uso de la tarjeta $\mathrm{BIP}^{5}$. Si bien los participantes no refirieron poseer dificultad en el uso de sus tarjetas de pago, emergió la estrategia anticipatoria de contar con dos tarjetas BIP, según lo referido por un participante: “... yo prefiero no andar con una tarjeta sino con dos... porque uno puede perderla o puede ser que te la roben...". En otros casos, el contar con una tarjeta extra, se refirió como una estrategia de adaptación en el caso que la tarjeta primaria no tuviese dinero cargado.

Por otra parte, se valoraba positivamente el uso de la tarjeta de pago debido a que permitía a los participantes salir de sus hogares sin dinero, lo que generaba la sensación de mayor seguridad ante eventuales robos, así como asaltos a los buses dado que los conductores no manejan dinero en efectivo.

Los participantes reconocían la frecuente necesidad de contar con el apoyo de otras personas, en especial en aspectos de movilidad y acceso al sistema de buses. El subir y descender del bus emergió como una dificultad, por ello tomaban medidas de precaución para evitar caídas, tales como descender por las puertas delanteras para contar con la

\footnotetext{
${ }^{5}$ La tarjeta BIP es un dispositivo electrónico de carga de dinero, la cual es la única forma de pago en el sistema de buses del transporte público. La recarga de dinero de esta tarjeta se realiza en cajas del metro de Santiago, o en sucursales específicas de carga BIP.
} 
atención del conductor del bus, o afirmarse constantemente de los pasamanos mientras se desplazaban y otras estrategias preparatorias según lo comentado: “...trato de andar con lo menos posible de cosas en las manos, para dejar las manos libres para sujetarme ...”.

\section{3. ¿Bus o metro? Razones para la elección}

Al analizar las entrevistas surgía de forma espontánea en los participantes la tendencia a comparar los dos dispositivos centrales del plan Transantiago: bus y metro. Los participantes exponían razones variadas que explicaban el por qué se prefería el uso de un dispositivo de movilidad por sobre el otro. Los participantes que optaban por utilizar el metro referían que era debido a la posibilidad que brinda éste de pagar un pasaje a menor costo, y también a la rapidez con que éste les permitía movilizarse a sus destinos. Por su parte, los participantes que preferían el uso de los buses lo hacían ya que percibían que éstos eran menos concurridos, los demás usuarios eran respetuosos con las personas mayores (evidenciándose, por ejemplo, en que liberaban los asientos preferenciales sin necesidad de que fuesen solicitados por ellos) y que los recorridos de los buses les eran de mayor utilidad en relación con la cercanía de los paraderos con los lugares que frecuentaban.

\section{Influencia del ambiente}

El ambiente físico emergió como un importante determinante del desempeño. La movilidad y acceso a los buses fue un tema de interés para los participantes como un elemento que dificultaba su desempeño. Surgieron en los relatos percepciones de incomodidad e inseguridad respecto de la infraestructura de los buses, por cuanto se reconocía en ella un aspecto de riesgo para sufrir caídas.

Los participantes que utilizaban los buses refirieron que pese a existir un espacio de detención establecido, los buses tendían a detenerse distantes de la acera, lo que aumentaba la diferencia en la altura entre ambas superficies, obligándoles a un mayor esfuerzo y percepción de riesgo al subir al bus. Uno de los participantes refirió: “...considero que los buses no, no son recomendables para nuestra edad, o sea esta clase de buses... otros buses que tengan las pisaderas más bajas, que se acerquen mucho a la acera porque o sino uno queda en el aire y se cae...". Pese a esto los participantes reconocían el beneficio del sistema de suspensión neumática auto regulado con el que se puede acomodar la altura del bus, según se refirió: “... en algunas ocasiones me ha tocado ver que los bajan un poquitito... así que ahí no tengo ningún problema...”.

Sobre las percepciones acerca del interior del bus, los participantes destacaban la incomodidad asociada con características de los asientos, como la inclinación acentuada sin apoyos, el material no antideslizante con que están confeccionados, y la disposición de sentarse mirando hacia atrás, uno de los participantes mencionó: “...porque uno se sienta y se resbala para todos lados, y cuando el bus para, de repente va a partir y se mueve así (se mueve bruscamente hacia delante), entonces si usted no está con las dos manos, a nosotros, las personas mayores nos zamarrea...". Por otro lado, los participantes percibían una mala amortiguación de los buses que genera movimientos bruscos y sorpresivos en el interior del bus, uno de ellos refirió: “...pero usted da los medios saltos, cada lomo de toro, cada hoyo que hay (...) no tiene amortiguación...”. 
En relación con la pantalla del validador de la tarjeta de pago en el interior de los buses, los participantes percibían que éste generaba dificultad para ver el saldo disponible, al respecto uno de los participantes refirió: “... cuando uno marca la tarjeta ahí le indica cuánto queda pero con los lentes yo no alcanzo a ver ahí cuánto quedaba...”.

Los participantes mencionaban que la altura de los pasamanos de los buses era excesiva y que su disposición no era óptima principalmente en los buses articulados, ya que para acceder a los asientos preferenciales, las personas mayores debían caminar sin apoyo por la tornamesa central en movimiento, al respecto uno de los participantes refirió : “... es incómodo, porque no hay cómo tomarse...yo soy muy bajo, entonces queda muy alto... al pasar uno para atrás no hay donde afirmarse y es una rueda como que se va moviendo que uno no puede pasar...".

En términos del funcionamiento del sistema de buses, los participantes percibían una desorganizada frecuencia, situación que generaba aglomeraciones al interior y fuera de ellos. Uno de los participantes refirió: "me ha tocado esperar no sé cuánto rato las micros (buses)... demora un montón, y si no, pasan dos seguidas bien rápido y se van...". Asimismo, reconocían como una dificultad personal el deber dirigirse caminando a los paraderos establecidos, refiriendo como ventaja comparativa el funcionamiento del sistema de buses anterior al actual en el que buses no tenían paraderos definidos. En relación con las rutas, se percibía que no habían sido consideradas las rutas del sistema de transporte anterior, refiriendo: “... debieran las autoridades y nuevos empresarios de reconocer que las rutas antiguas, eran fruto de una larga experiencia, (las autoridades y nuevos empresarios) conocían los recorridos que necesitábamos los pasajeros para ir a trabajar, a los hospitales, en la forma más corta y económica...".

Por último, el ambiente social, es decir, las actitudes y características del resto de los usuarios del sistema, emergió también como un tema relevante para el desempeño. En el uso de buses, los participantes percibían que recibían ayuda por parte de terceros, y de modo global expresaron opiniones positivas en relación con la disposición tanto de conductores como del resto de los usuarios, para ayudarles en caso de requerirlo.

En contraste con las dificultades para el desempeño asociadas con el funcionamiento de los buses, los participantes se encontraban conformes con los horarios de funcionamiento del metro: “...bueno el metro, funciona muy bien porque festivo y, no festivos siempre está funcionando y pasando distintos trenes rápido...". Los participantes destacaban la existencia del beneficio tarifario para adultos mayores propio del metro, no obstante consideraban que el acceso a esta información ocurría a través de medios informales a partir de otros amigos o familiares, por lo que muchos adultos mayores desconocían esta posibilidad.

Sobre el ambiente físico del metro, los participantes destacaban el uso de escaleras mecánicas como elemento que facilitaba su acceso a las estaciones. Emergió como un factor que dificultaba el desempeño en el metro la gran cantidad de gente que ingresaba en sus vagones, lo que dificultaba su posibilidad de utilizar los pasamanos y de acceder a la información visual, por ende, valoraban la existencia de anuncios por altoparlante que les 
permitía anticipar su descenso en las estaciones destino. La excesiva cantidad de usuarios emergió también como un factor de preferencia por usar el bus sobre éste, respecto a esto un participante refirió: "...y eso de andar en el metro, como animales cuando los llevan en esos camiones grandes al matadero, a la gente así en el metro, más o menos a las nueve o diez... es tremendo...me da miedo, mucha gente se junta en los andenes del metro, es tremendo...".

La percepción de seguridad en el metro era mayor en relación a la posibilidad de sufrir un eventual robo, atribuido principalmente a la existencia de funcionarios que supervisan los andenes, y a la menor posibilidad que existía de que la gente subiera sin pagar, uno de los participantes refirió: “...cuando uno está sacando boleto, ellos (funcionarios del metro) están ahí también observando a todo el público, esos también son necesarios porque los ladrones no pueden entrar a robar...".

Vale la pena mencionar que ninguna de las personas mayores entrevistadas había sufrido accidentes en el interior del metro. A pesar de esto, los participantes refirieron de manera global sentirse inseguros en relación con factores ambientales de su uso, expresándose en temor a sufrir robos, a perder estabilidad por la inercia y caer, y a no poder descender del metro por la elevada congestión usuarios en su interior.

\section{Significado del uso: autonomía versus exclusión}

Este tema destaca el valor personal que tenía el uso de transporte público para los participantes.

Un aspecto del significado se relacionó con la relevancia otorgada a la percepción de autovalencia como base para seguir utilizando el sistema de transporte, y de este modo mantener en vigencia su participación independiente en actividades significativas que los auto definían, al respecto uno de los participantes refirió: “....si uso el transporte estoy vigente, sigo siendo yo mismo... con eso le respondo...”. El uso de transporte público era entonces considerado como indicador de autonomía y de continuidad biográfica.

Los participantes refirieron la relevancia que el uso del transporte público tenía en su participación social. Reconocían que algunas características del sistema, al no considerar las diversidad funcional que el grupo etario puede llegar a tener, colabora con la marginación de personas que se encuentran en un mayor estado de dependencia: “...si los buses fueran de mejor manera hechos, de mejor calidad, podría decirlo más cómodo para la gente, sobre todo para los adultos mayores porque, es que como que nos han dejado fuera (...) se están olvidando de nosotros, y si llego a usar bastón tal vez ya no las tome, me da cosa, ¿me entiende? pero mientras tanto, todavía sirven, pero no para todos, y por el resto de las señoras ahí me da pena, porque quedan, todas quedaron afuera, todas quedaron afuera...".

De manera global emergió la necesidad de modificar las características físicas de los dispositivos metro y bus para facilitar el desempeño de la población mayor: “...por mí yo lo haría de nuevo, haría buses especiales para el adulto mayor, como hay en otras partes". Destacó la percepción que al ser personas mayores, sus reclamos o sugerencias no serían validadas por quienes administran el sistema, aspecto que perpetuaría su sensación de marginación; Al respecto uno de los participantes comentó: “... a mí me gustaría escribir 
todo esto al transporte pero no creo que me escuchen, ni que me vean, ni me lean, ni tomen en cuenta...".

\section{DISCUSIÓN}

El objetivo general de este trabajo fue explorar la percepción de las personas mayores sobre su desempeño en el uso del transporte público, con miras a generar información más específica sobre las características de esta actividad en adultos mayores chilenos y los factores personales, ambientales y propios de la ocupación involucrados en ésta. A través de los relatos obtenidos de esta investigación ha sido posible conocer distintas condiciones relacionadas con los tres aspectos mencionados.

Uno de los aportes primarios de este trabajo se relaciona con la posibilidad de conocer la función del uso de transporte en el grupo de personas mayores participantes. Esta actividad emerge como una ocupación que vincula al individuo con su medio ambiente social, y que le permite mantener el sentido personal ya que posibilita la realización de otras actividades instrumentales o de participación social. Esta información es de utilidad en la práctica de Terapia Ocupacional, ya que posiciona el uso de transporte público como un objeto relevante de evaluación y de intervención si se busca la promoción de la participación en ocupaciones.

Otro aspecto de aporte a la práctica de Terapia Ocupacional, se relaciona con el conocer mecanismos anticipatorios o preparatorios para un mejor uso del transporte público. En función de la importancia que atribuyen los participantes a continuar el desempeño en esta ocupación a pesar de las limitantes del medio y de las características personales, se concluye que las personas mayores pueden desplegar habilidades adaptativas, desarrollando hábitos y estrategias para continuar usando el transporte público de forma segura. El sentirse eficaces en el desempeño de esta ocupación y por tanto capaces de concretar sus intereses, motiva a los entrevistados a realizar las adaptaciones que sean necesarias para mantener un equilibrio entre las capacidades personales y las demandas ambientales.

Dichas adaptaciones se configuran en rutinas rigurosas y estructuradas en cuanto a la forma con que utilizan el sistema, las cuales aparecen sistemáticamente en la vida de los participantes en respuesta a la percepción de inseguridad. Del mismo modo, podría considerarse que el grupo de participantes, al ser totalmente autónomos e independientes en el uso del transporte, ha desarrollado estrategias de adaptación "exitosas" que pueden ser útiles de considerar en los contextos de intervención. En esta línea puede mencionarse por ejemplo, la preparación por medio del acceso a información y el conocimiento de las rutas frecuentes, como una estrategia cotidiana que podría aportar a grupos vulnerables o con dificultad en la elaboración propia de estas estrategias.

El ambiente físico de desempeño, una frecuente área de interés en la investigación y práctica de la Terapia Ocupacional, es coherentemente un componente relevante en las experiencias de las personas mayores en el uso del transporte público. Por medio de este trabajo se logra visualizar oportunidades concretas de mejoramiento de los dispositivos físicos de movilización, como buses y metros, que pueden aportar a una mayor percepción de seguridad e independencia de las personas mayores chilenas en el uso del transporte. Por 
mencionar algunas, la entrega de información por medio de distintos canales (ejemplo, visual y auditivo), la reducción del esfuerzo físico por medio de la adecuación de la altura del acceso a los buses en relación con la acera, la modificación de la altura y tipos de pasamanos, entre otros, son factores del ambiente físico que pueden incidir en una mejor experiencia durante la actividad.

Las personas mayores participantes perciben que el sistema de transporte público no está diseñado de acuerdo a sus necesidades y dificultades, por lo que el ambiente físico puede comportarse como facilitador o limitante del desempeño, de acuerdo a cómo sus demandas interaccionan con las capacidades personales. Para un desempeño satisfactorio y seguro de esta actividad, la persona mayor requiere que los espacios públicos y los medios de transporte sean accesibles considerando las limitaciones que en general les afectan (Marsden, Cattan, Jopson \& Woodward, 2008). En la ciudad de Santiago se observa cómo el transporte público ha sufrido modificaciones en los últimos años, vinculados principalmente a la modernización de la infraestructura de los buses, que buscó una mayor accesibilidad y adaptación a los requerimientos de las personas con necesidades especiales. Sin embargo, siguen existiendo barreras ambientales importantes para el grupo de personas mayores, configurándose en ocasiones como elementos que dificultan el desempeño.

Ramos \& Nazif (2005) concluyeron que las personas mayores se sentían vulnerables respecto a actitudes de conductores de bus y pasajeros, vivenciando situaciones que ponían en riesgo su integridad física y emocional, lo que generaba una escasa sensación de protección. En contraste, lo analizado a partir de este estudio destaca que el ambiente social de desempeño es percibido por las personas mayores entrevistadas como favorecedor del desempeño. Esto podría reflejar un cambio de actitud positivo hacia nuestra población mayor.

A partir de esta investigación es posible también reflexionar sobre el significado de la actividad, un aspecto no explícito de la ocupación, que la condiciona de manera transversal y subjetiva. En el grupo de participantes, el uso de transporte público era percibido como un indicador de autonomía y de mantención de la identidad personal, dado que posibilita la mantención de otras actividades significativas. Este hallazgo es similar al reportado en población mayor en un estudio realizado por Mardsen et al. (2010) que buscó detectar necesidades de las personas mayores en el uso del transporte público en Inglaterra, el cual destaca la importancia para las personas de estar capacitadas para utilizar el transporte público en pos de mantener conexiones con familiares, amigos y vecinos, y mantener la independencia como un elemento crítico de bienestar. Del mismo modo, de este estudio se desprendió la importancia de contar con la habilidad para llevar a cabo esta actividad, que se vincula con la posibilidad de continuar la realización de actividades de otras áreas significativas. En el mismo estudio, se concluyó que existe una evidencia sustancial de que las personas mayores sufren más que la mayoría por la calidad del transporte público, principalmente por su infraestructura, percibiendo que el sistema de transporte estaba diseñado para personas sanas y no para las que son frágiles. Lo referido es entonces coincidente con los hallazgos de nuestro trabajo que describen la vulnerabilidad percibida ante el ambiente físico de desempeño, en especial para aquella persona que requiera algún tipo de apoyo o que presente algún grado de dependencia. 
Desde una mirada de la metodología usada, las entrevistas realizadas y su análisis interpretativo permitieron la obtención de hallazgos subjetivos relacionados con las percepciones de los adultos mayores, contribuyendo al logro de los objetivos de este estudio. Durante el diseño de este trabajo, en el equipo investigador se planteó la pertinencia de utilizar otra técnica de recogida de datos por medio de observación con el fin de obtener la información del fenómeno a estudiar desde el contexto donde ocurría. No obstante, se consideró que al tratarse de una temática escasamente abordada en nuestro país, se carecería de información para definir los aspectos prioritarios a observar in situ, favoreciendo un sesgo interpretativo en los hallazgos (Punset, 2006). Dado que este estudio vislumbra temas principales desde una perspectiva exploratoria, podría considerarse la metodología observacional en trabajos posteriores que permitan profundizar o probar nuevas hipótesis sobre el fenómeno estudiado en este trabajo (Rodríguez, Gil, \& García 1996). Del mismo modo, este estudio no intentó establecer diferencias según otras variables, como tramo etario y género, que pueden resultar de interés posterior, especialmente dada la asociación de la edad avanzada con mayores niveles de dependencia (Kane, Ouslander,\& Abrass, 2000).

Los hallazgos provenientes de esta investigación representan una aproximación a las experiencias reales de nuestros adultos mayores al interactuar con el sistema de transporte público de la ciudad de Santiago. Finalmente, es el propósito de este trabajo colaborar con un sistema de transporte que si bien ha desarrollado un avance que puede ser favorable para la población general, presenta oportunidades de mejora para aportar a que las personas mayores perciban una mejor experiencia en su uso y se promueva de mejor forma su participación en sociedad.

\section{Agradecimientos}

Este trabajo ha sido realizado gracias al apoyo del equipo multiprofesional de la Sección de Geriatría del Hospital Clínico Universidad de Chile, y del establecimiento de larga estadía Fundación Villa Padre Alberto Hurtado quienes posibilitaron contar con las valiosas experiencias de los y las participantes de nuestro estudio. A estos últimos, agradecemos su disposición a abrir las puertas de su hogar y de sus experiencias.

\section{REFERENCIAS BIBLIOGRÁFICAS}

- Aristizábal, C. (2008). Teoría y metodología de la investigación. Recuperado el 14 de Julio de 2011 desde http://www.funlam.edu.co/administracion.modulo/NIVEL06/TeoriaYMetodologiaDeLaInvestigacion.pdf

- Asociación Americana de Terapia Ocupacional (AOTA). (2008). Marco de Trabajo para la Práctica de la Terapia Ocupacional: Dominio y Proceso. Recuperado el 13 de abril de 2012 desde http://www.terapiaocupacional.com 
- Duarte, P., Pedro, P. (2010). Terapia ocupacional en geriatría: principios y práctica. (Tercera edición). Barcelona: Editorial Elsevier Masson; 351-361.

- Huber, G., Gürtler, L. (2008). Manual de programa para analizar datos cualitativos. Recuperado el 9 de Octubre de 2011, desde http://www.aquad.de/spa/m-cap05.pdf

- Instituto nacional de estadísticas de Chile. (2002). Índice general Censo 2002: Resultados. Recuperado el 13 de Octubre de 2010, de: http://www.ine.cl/canales/usuarios/cedoc_online/censos/pdf/censo_2002_volumen _II.pdf

- Kane, R., Ouslander, J., \& Abrass, I. (2000). Geriatría Clínica. (Cuarta Edición). México: Editorial McGraw-Hill Interamericana.

- Kielhofner, G. (2006). Fundamentos Conceptuales de la Terapia Ocupacional (Tercera Edición). Buenos Aires, Argentina: Editorial Médica Panamericana.

- Marsden, G., Cattan, M., Jopson, A., \& Woodward, J. (2008). Older People and Transport: integrating transport planning tools and user needs. Strategic promotion of ageing research capacity. Reino Unido: Ediciones University of Leeds. Recuperado el 16 de Octubre de 2010, desde http://www.sparc.ac.uk/media/downloads/executivesummaries/exec_summary_ma rsden.pdf

- Ministerio de Planificación de Chile. (2006). Encuesta de Caracterización Socioeconómica Nacional: Resultados Adulto Mayor. Recuperado el 15 de noviembre 2012, de:

http://www.ministeriodesarrollosocial.gob.cl/casen/publicaciones/2006/Resultados_ Adulto_Mayor_Casen_2006.pdf

- Ministerio de Transportes y Telecomunicaciones.(2012). Transantiago informa. Recuperado el 13 de Octubre de 2010 desde

- http://www.transantiago.cl/QUIENESSOMOS/HISTORIA/index.htm

- Punset, E. (2006). El viaje a la felicidad: las nuevas claves científicas. (Octava edición) Recuperado el 24 de noviembre de 2011, desde http://www.quedelibros.com/libro/44676/El-viaje-a-la-felicidad.html

- Ramos, M., Nazif, I. (2005). Adultos Mayores: Su interacción en el sistema de transporte terrestre. Instituto de Normalización Previsional de Chile. Recuperado desde http://www.conaset.cl/cms_conaset/archivos/Estudio $\% 20$ conductual $\% 20$ adulto $\% 2$ 0mayor.pdf 
- Rodríguez, G., Gil, J., \& García, E. (1996). Metodología de la Investigación Cualitativa. Recuperado el 14 de Julio de 2011, desde

http://tecnoeduka.110mb.com/documentos/investiga/articulos/rodriguez $\% 20$ gil $\% 2$ 0garcia\%20cap3.pdf

- Servicio Nacional del Adulto Mayor. (2009). Estudio Nacional de la dependencia en personas mayores. Recuperado el 15 diciembre 2012 desde http://www.senama.cl/archivos/estudiodependencia.pdf

- US transportation research Board Council. (1998). Transportation in an Ageing Society: improving mobility and safety for older persons. Special report 218. Recuperado el 23 de abril de 2012 desde: http://onlinepubs.trb.org/onlinepubs/conf/reports/cp_27.pdf

- Vargas, L. (1994). Sobre el concepto de percepción. Recuperado el 10 de Mayo de 2011, desde http://www.uam-antropologia.info/alteridades/alt8-4-vargas.pdf

- Wallace, R., Franc, D. (1999). Literature Review of the Status of Research on the Transportation and Mobility Needs of Older Women. Estados Unidos. Recuperado el 16 de Octubre de 2010 desde http://www.nhtsa.gov/people/injury/olddrive/nscrpt.html 